\title{
Juventud Rural: factores que influyen en el desarrollo de la actividad agropecuaria. Reflexiones sobre el caso argentino, ${ }^{1,2}$
}

\author{
Marcelo Sili ${ }^{3}$, Sandra Fachelli ${ }^{4}$ y Andres Meiller ${ }^{5}$
}

Resumen: El éxodo o la permanencia de los jóvenes en el medio rural es un tema sumamente discutido en América Latina. Este trabajo pretende contribuir a esta discusión sobre la base de una investigación empírica realizada a 400 jóvenes en Argentina, en la cual se muestran cómo influyen diferentes variables en el mantenimiento de los jóvenes en el medio rural realizando actividades agropecuarias. La hipótesis que se plantea y que finalmente se valida es que la pertenencia a familias productoras agropecuarias y la continuidad de estudios vinculados a la actividad agropecuaria son los factores claves que permiten mantener a los jóvenes en el medio rural realizando actividades agropecuarias. Las técnicas estadísticas utilizadas para realizar el análisis descriptivo son tablas de contingencia y comparación de medias, en tanto la regresión logística binaria múltiple se utiliza para realizar el análisis de dependencia y validar la hipótesis planteada.

Palabras claves: Juventud rural; Éxodo rural; Argentina; Desarrollo rural.

Abstract: The exodus or the permanence of young people in rural areas is a highly debated topic in Latin America. This work aims to contribute to this discussion based on an empirical research with a survey of 400 young people in Argentina. This study shows how different variables influence in keeping young people in rural areas engaging in agriculture activities. The hypothesis that arises and that is finally validated is that

1. Esta investigación fue desarrollada en el marco del Proyecto "Análisis de las problemáticas de la juventud rural e identificación de estrategias e instrumentos de generación de empleo y acceso a la tierra rural" financiado por la Unidad para el Cambio Rural del Ministerio de Agricultura, Ganadería y Pesca de la Nación y por la Universidad Nacional de San Martín. El estudio fue coordinado por Marcelo Sili y Juan Sanguinetti.

2. Data de submissão: 17 de fevereiro de 2016. Data de aceite: 15 de julho de 2016.

3. Universidad Nacional del Sur. Bahía Blanca, Argentina. E-mail: sili.marcelo@gmail.com

4. Universitat Atuònoma de Barcelona y Universitat de Barcelona. Barcelona, Cataluña, España. E-mail: Sandra.Fachelli@ub.edu

5. Nexos Economía y Desarrollo. Ciudad de Buenos Aires, Argentina. E-mail: andresmeiller@gmail.com 
belonging to farming families and studies related to agricultural activity are the key factors that allow young people to settle in rural areas and engage in agriculture. The statistical techniques used for descriptive analysis are contingency tables and comparison of means, while the multiple binary logistic regressions are used to perform dependency analysis and validate the hypothesis.

Key-words: Rural youth; Rural exodus; Argentina; Rural development.

DOI - http://dx.doi.org/10.1590/1234-56781806-94790540403

Classificação JEL: O18.

\section{Introducción}

Durante los últimos treinta años se produjo en Argentina un cambio estructural en la organización y la dinámica de los territorios rurales, que implicó una fuerte reducción y desintegración de la pequeña y mediana agricultura, y la consolidación de una nueva clase agraria más concentrada. Este proceso de modernización excluyente se caracterizó por la puesta en marcha de políticas de ajuste y desregulación, la desaparición de gran parte de los instrumentos tradicionales de política pública y la modernización tecnológica en los sistemas de producción agropecuarios (SILI, 2015). El conjunto de políticas implementadas impactó sobre las infraestructuras, los equipamientos y los servicios en las áreas rurales (cierre de ramales ferroviarios, reorganización administrativa, etc.) y en las empresas agropecuarias, las cuales se vieron obligadas a aumentar las escalas productivas, a mejorar los sistemas de gestión y a modernizar los sistemas de producción de manera que pudieran encontrar un nuevo punto de equilibrio y rentabilidad que les permitiera continuar sus actividades. Se privilegió así la expansión agraria y la rentabilidad de las grandes empresas, que pueden continuar su proceso expansivo incorporando capital y nuevas tierras, en detrimento del sector productivo familiar de todo el país. Ambos procesos son obviamente complementa- rios y se acompañan de otros fenómenos como la modificación en las estructuras agrarias, la concentración de la tierra y la reestructuración de los territorios rurales con la desaparición de cientos de pueblos, el abandono y despoblamiento del campo y la creciente concentración urbana, especialmente en las capitales de provincia y ciudades de importancia provincial.

Todos estos fenómenos han sido reiteradamente analizados en los últimos años desde múltiples espacios de investigación y desarrollo. Un elemento novedoso es que en todas las investigaciones recientes la preocupación por la organización y las dinámicas de los territorios comienzan a emerger como un tema clave. Así, de la histórica preocupación por la cuestión agropecuaria, tema clave en un país que históricamente dependió en gran medida de lo que sucede en sus áreas rurales, se fue pasando lentamente a una creciente preocupación por los problemas sociales y ambientales, donde el éxodo rural, las infraestructuras, la pobreza, la degradación ambiental, la pérdida de identidad, entre otras cosas, cobran cada vez más importancia en la agenda pública. Dentro de este contexto de transformación rural la problemática de la juventud rural, ha sido durante muchos años un tema de escaso interés en América Latina (DURSTON, 1998), y ahora empieza a emerger como un factor clave para el futuro rural. Los crecientes estudios realizados 
coinciden en señalar que las juventudes rurales constituyen un sujeto múltiple, heterogéneo y altamente complejo, con recursos, dinamismo y potencialidades muy diferentes según las sociedades, las particularidades de cada territorio, la dimensión y el tipo de tenencia de la tierra, los sistemas productivos predominantes, la posición social y el género (DURSTON, 1998; KESSLER, 2005; SILI, 2005a, CASTRO et al., 2015).

Estos estudios han permitido un avance significativo en el análisis y el conocimiento de la juventud rural y en señalar sus especificidades que lo diferencian respecto de la juventud urbana, destacando en los jóvenes rurales la preponderancia de las relaciones familiares patriarcales (WELLER, 2006), una centralidad en torno a la problemática de la tierra (DIRVEN, 2002; SILI, 2011), la existencia en muchos casos de la pluriactividad como una forma de subsistir por la insuficiencia de los ingresos provenientes del trabajo agrícola familiar (WELLER, 2006; MAURICIO, 2011), y la presencia de tensiones identitarias entre lo local y lo global.

Sin embargo un tema clave en torno a la juventud rural en este contexto de cambios estructurales del mundo rural ha sido el dilema que se le plantea a los jóvenes entre permanecer en las áreas rurales o migrar a otras ciudades en busca de oportunidades de estudio o trabajo que les permita mejorar sus condiciones de vida actuales y sobre todo construir un proyecto de vida que ellos visualizan como más venturoso que en las zonas rurales donde habitan (DURSTON, 1998; KESSLER, 2005; SILI, 2005b; SILI 2007; COSTA, 2013; GOMES y CARVALHO, 2015). Resulta claro que la permanencia de los jóvenes en las zonas rurales o el éxodo hacia otras zonas o ciudades es una situación compleja que depende de numerosas variables, y que no se manifiesta en todos los jóvenes y todos los territorios rurales por igual (DURSTON 1998). Sin embargo, el éxodo aparece como la solución más clara y viable para construir un proyecto de vida más promisorio.

El objetivo de este trabajo es identificar y analizar cuáles son los factores que potencian y favorecen la permanencia de los jóvenes en las áreas rurales, desarrollando allí actividades agropecuarias. Entender estos factores no es sólo una preocupación de índole académico, al mismo tiempo, estos conocimientos son claves en una política nacional de desarrollo agropecuario y rural pues permitiría, a través de acciones específicas, fortalecer los factores claves de arraigo de los jóvenes en el medio rural y garantizar la sucesión y la continuidad de las explotaciones agropecuarias, especialmente pequeñas y medianas, evitando así, tal como lo plantea Abramovay un "esvaziamento do campo" que es "uma das maiores ameaças para o desenvolvimento rural" (apud COSTA y RALISCH, 2013, p. 416).

\section{Marco conceptual de referencia e hipótesis de trabajo}

El análisis del éxodo o permanencia de los jóvenes constituye un tema de análisis cada vez más importante a nivel global y en América Latina, no solamente por tratarse de una problemática que afecta a la dinámica rural actual, sino y sobre todo por las perspectivas de evolución del mundo rural y de los sistemas agroalimentarios hacia el futuro, pues es la población joven la responsable de organizar y dinamizar las áreas rurales en las próximas décadas (WHITE, 2012). De esta manera, el análisis de la juventud hay que situarlo en un contexto temporal y dinámico y no sólo en términos de impactos territoriales actuales.

Los estudios en torno a esta problemática responden actualmente a dos grandes líneas de reflexión.

Una primera línea de reflexión busca comprender los fenómenos migratorios o al contrario, el arraigo de los jóvenes a sus territorios rurales a partir del análisis de las dinámicas sociales y culturales de los jóvenes. Tres temas claves se analizan desde esta corriente de reflexión:

1. La primera es el nivel educativo de los jóvenes rurales y su relación con la permanencia y la migración. Existe un gran diversidad de estudios que analizan esta 
relación, gran parte de ellos señalan la existencia de una relación directa entre aumento de los niveles educativos y propensión a migrar hacia otros lugares (KESSLER, 2005; BECERRA, 2006; COTA MENDONÇA, 2013; CASTRO et al., 2015). Otros autores señalan ciertas especificidades vinculadas especialmente con los tipos de educación, aduciendo que no se pueden plantear generalidades, sino que al contrario, es necesario ver en detalle el tipo de formación de los jóvenes. Así, White (2012) y Dirven (1995) por ejemplo señalan que la educación agropecuaria, especialmente aquella que se combina con la realización de trabajos agrícolas, es el mejor camino para evitar la destrucción de las habilidades de los jóvenes rurales en materia de producción agropecuaria, permitiendo la permanencia de los mismos en las áreas rurales y en el sector agropecuario.

2. El segundo tema clave dentro de esta línea de reflexión, es el peso que tiene la identidad rural y agraria de los jóvenes y su rol en la construcción del arraigo a las áreas rurales. La identidad rural esta entendida según los planteos de Sili (2005b), Theobald y Wood (1992) y Creed y Ching (1997) como la autoidentificación o sentimiento de pertenencia de los jóvenes a un tipo de territorio (los territorios rurales) y a un tipo predominante de actividad (la actividad agropecuaria). Es claro que la identidad de los jóvenes es muy cambiante dada la nueva lógica de relación campo ciudad, el fuerte desarrollo de los transportes y comunicaciones y la construcción de una nueva cultura global (KESSLER, 2005; JURADO, 2012). A pesar de las nuevas condiciones generadas por este cambio tecnológico y la globalización, "la identidad de las personas jóvenes rurales sigue ligada al territorio rural, en escenarios micro tales como la familia, hasta escenarios meso tales como el municipio" (JURADO, 2012, p. 68). Esta identidad rural que los jóvenes van construyendo a partir de su primera infancia se realiza, más allá del tipo de educación recibida, a partir de una doble vía: a) por el tipo de lugar donde habitan los jóvenes, ya sea en los pueblos o en el campo (SILI, 2015); b) por el tipo de actividad que realizan en su familia, generalmente dedicadas a actividades agropecuarias (DIRVEN, 1995). Más allá de las condiciones en las cuales se construye la identidad rural, que puede conformarse en un valor social y territorial muy importante, una premisa importante que se repite en estos autores es que el ambiente de socialización es estructurante de la identidad y tiene vital importancia en la construcción de la personalidad y la trayectoria biográfica de los jóvenes (JURADO, 2012). Es en función de ello que la dimensión identidad rural, si bien es un tema poco analizado, debe ser considerado con mucha mayor profundidad e importancia a la hora de entender las decisiones de los jóvenes frente a su futuro.

3. El tercer tema de análisis son las preferencias por el modo de vida rural. Diversos autores analizan las preferencias que los jóvenes rurales manifiestan por vivir en el medio rural, preferencias que tal como lo señala Dirven "está asociada a la valoración dada a los lazos de parentesco y de compadrazgo, las tradiciones culturales, las formas de ayuda mutua y las posibilidades de lograr una posición de respetabilidad en la comunidad" (DIRVEN, 1995, p. 125). La autora también señala que "las evidencias cualitativas sugieren que un número importante de jóvenes, sobre todo varones, muestran preferencia por la agricultura por sobre otras alternativas, siempre y cuando esta actividad les permita acumular lo suficiente para no vivir en la pobreza" (DIRVEN, 1995, p. 125). En su estudio sobre jóvenes rurales de 
Estados Unidos, Bajema (2002) coincide también en el peso que tiene la vida comunitaria y familiar, y las tradiciones, agregando también la preferencia que los jóvenes hacen por vivir en un espacio más sano desde el punto de vista ambiental. Estas evidencias empíricas también se verifican en el caso de Brasil, donde se observa que más del $70 \%$ de los jóvenes encuestados de Florestópolis piensan en continuar en el mundo rural (COSTA y RALISCH, 2013).

Una segunda línea de reflexión sobre la cual sustentamos esta investigación está centrada en la dimensión económica productiva y la posesión por parte de los jóvenes de factores de la producción. En esta línea de reflexión aparecen dos temas claves de análisis:

1. El primer tema clave es el vínculo de los jóvenes con la tierra. Así, una gran cantidad de autores centran su análisis en torno a la posesión de tierras y la problemática de la sucesión familiar. Diversos trabajos dan cuenta sobre las dificultades de los jóvenes para acceder a la tierra, situando principalmente el problema en las nuevas dinámicas de concentración por parte de los sectores más empresariales (SILI, 2005b; WHITE, 2012), la carencia de políticas públicas capaces de promover el acceso a la tierra por parte de los jóvenes (WHITE, 2012) y las dificultades de los procesos sucesorios de la tierra dentro del ámbito familiar (DURSTON, 1998; DIRVEN, 2002; GOMES y CARVALHO, 2015). En principio, para el conjunto de estos autores, la dificultad para acceder a la tierra se explicaría en gran parte por la migración de los jóvenes de las áreas rurales. No obstante también existen estudios y análisis que muestran la existencia de márgenes de maniobra para acceder a la tierra, tal como lo señala Dirven "Aún para los jóvenes sin tierra, existe alguna posibilidad de acceder a ella a través del matrimonio con una muchacha que hereda, a través del acceso compartido a tierras de un hermano, cuñado $u$ otro familiar, o a través del arriendo o de la mediería" (DIRVEN, 1995, p. 125). Otros estudios y reflexiones sobre la problemática rural especialmente las corrientes de pensamiento ligadas a la nueva ruralidad en América Latina, ponen menos énfasis en el acceso a la tierra como un factor clave de permanencia en las áreas rurales, otorgándoles más importancia a la innovación y la dimensión cultural e identitaria (LINCK, 2001; SCHEJTMAN y BERDEGUÉ, 2003; GARRABOU et al., 2014). Más allá de estas diferentes posiciones, consideramos que la tenencia de la tierra segura es un factor que no puede ser soslayado en los estudios sobre la juventud rural, tal como lo demuestran los trabajos de White (2012) y Sili (2015).

2. El segundo tema de reflexión es la relación entre los jóvenes y el empleo, especialmente empleos formales $y$ bien remunerados que les permitan permanecer en las áreas rurales en igualdad de condiciones que en otros lugares. Los diversos estudios sobre esta problemática plantean la existencia de dificultades estructurales para acceder al empleo en las zonas rurales por parte de los jóvenes debido a su falta de experiencia o de formación, o a la falta de empresas o unidades productivas con capacidades reales de generar empleos formales de calidad. Esta situación se ve agravada por la carencia de políticas locales capaces de fomentar y sostener la generación de empleo rural, generalmente en ámbitos rurales con municipios o gobiernos muy débiles o con bajas capacidades organizacionales (SILVA, 2002; KESSLER, 2005; MAURICIO, 2011; WHITE, 2012). Los diversos estudios sobre la juventud rural señalan como tema importante las grandes oportunidades que tendrían los 
jóvenes hacia el futuro dada su mayor capacidad y destreza con herramientas tecnológicas modernas, lo cual les permitiría no sólo acceder a empleos agropecuarios de mayor complejidad (manejo de maquinaria compleja), como también a empleos rurales no agropecuarios vinculados a los servicios y que en muchos casos pueden realizarse en forma remota (DIRVEN, 1995; PACHECO LADRÓN DE GUEVARA, 1999; MAURIZIO, 2011; SILI, 2015).

Complementando estas nociones, se debe aclarar que este trabajo diferencia el significado de lo rural y lo agropecuario. Lo rural hace referencia a un tipo de territorio con características específicas (baja densidad, fuerte relación con los recursos naturales, relaciones directas entre las personas, predominio de actividad agro-silvopastoriles, etc.), en tanto lo agropecuario hace referencia a un tipo de actividad económico productiva como es la producción ganadera, la agricultura y la silvicultura, por lo tanto no deben considerarse como sinónimos. Lo rural es un tipo de territorio, en tanto lo agropecuario es un tipo de actividad productiva, que claramente tiene lugar en los territorios rurales, pero no explica totalmente el funcionamiento de este tipo de territorios (SILI, 2015).

Todas estas bases argumentales y estos antecedentes empíricos y conceptuales, nos permiten plantear una hipótesis en relación a los factores que impulsan o limitan la permanencia de los jóvenes en las áreas rurales desarrollando actividades agropecuarias propias ${ }^{6}$. Así la hipótesis plantea que el hecho de pertenecer a una familia productora agropecuaria y el haber realizado o realizar estudios agropecuarios influye positivamente en que el joven desarrolle una actividad agropecuaria propia.

6. Se entiende que el joven desarrolla una actividad agropecuaria propia cuando participa activamente (solo o en sociedad) en las decisiones técnico-productivas y económicas-financieras de una explotación o emprendimiento agropecuario.

\section{Modelo de análisis y metodología}

Para poder corroborar la hipótesis de este trabajo, mencionada en el apartado anterior, se plantea el modelo de análisis que se presenta en la Figura 1.

El desarrollo de actividad agropecuaria propia es la variable a explicar y para ello se utiliza un conjunto de variables independientes que la literatura ha mostrado que tiene influencia, y que entonces queremos corroborar en el ámbito geográfico concreto en el que hemos llevado a cabo el estudio.

Así, en primer lugar nos proponemos contrastar si proceder de una familia productora agropecuaria y realizar o haber realizado estudios agropecuarios ${ }^{7}$ influye en el hecho de que el joven realice una actividad agropecuaria propia. Esta última variable, es dicotómica y distingue entre los jóvenes que desarrollan en el momento de la encuesta una actividad agropecuaria propia (16\% del total de jóvenes) y aquellos que no (aunque deseen o no hacerlo).

Con el fin de testar la hipótesis contamos con algunas variables de control que nos permitirán interpretar mejor la influencia de las variables independientes. Las variables de control utilizadas son las siguientes: sexo, edad, ubicación de la vivienda (ámbito rural agrupado que incluye pueblos de hasta 10.000 habitantes, o ámbito rural disperso, que hace referencia a personas que habitan en las explotaciones agropecuarias o en parajes rurales de muy baja densidad de población), nivel de estudios (bajo -hasta primario completo-, medio -hasta secundario completo- o alto -terciario y universitario completo e incompleto-), cantidad de hermanos, ingreso total del joven (clasificado en tres categorías: Hasta $\$ 5.000$, más de $\$ 5000$, o no declara ingresos $\left.{ }^{8}\right)$, pertenencia a una familia

7. Esta variable se refiere a la realización de estudios agropecuarios en el pasado o en la actualidad. De la totalidad de jóvenes que han realizado estudios agropecuarios, el 31\% se encuentra cursándolos al momento de la encuesta, y el $69 \%$ restante los ha realizado en el pasado.

8. Al momento de realizar la encuesta (enero y febrero del 2014), \$5.000 eran equivalentes a u\$s 670. 
Figura 1. Modelo de análisis propuesto

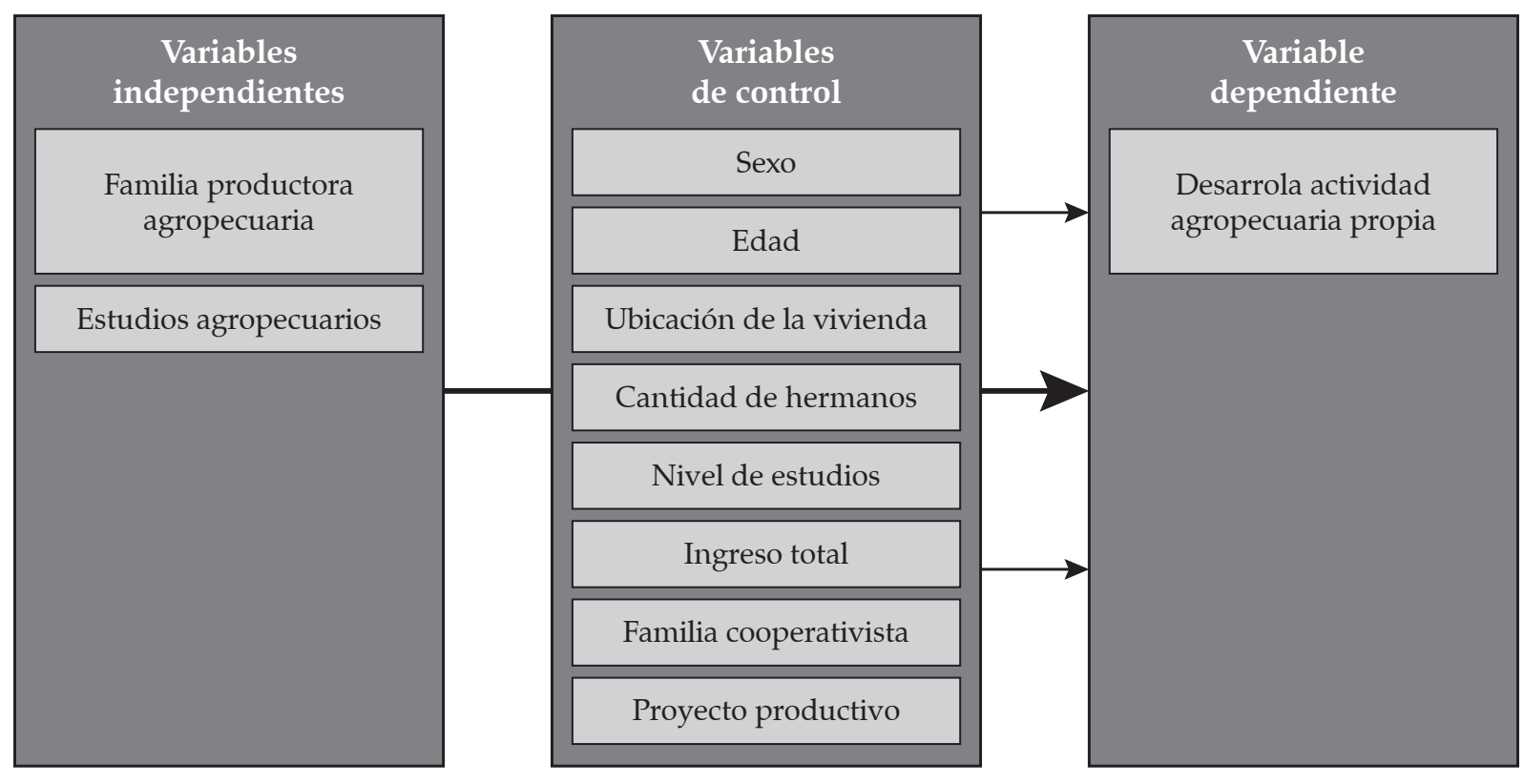

Fuente: Elaboración propia.

donde hay miembros adheridos a una cooperativa rural (sí, no, o con alguna relación comercial), y disposición a realizar un proyecto productivo propio en el mediano o largo plazo (sí o no).

Por su parte, una nota importante sobre la variable sexo es que en este análisis no abordamos cuestiones vinculadas al género, que son sumamente importantes de tener en cuenta, como los mecanismos de herencia o traspaso de explotaciones/propiedades agrarias hacia el varón, el control social y parental de la mujer a la que se sigue recluyendo al ámbito de lo privado casa familiar- y a trabajos y roles relacionados con su papel de madre y esposa o relacionados con el cuidado de familiares mayores, entre otros. Todos estos factores son más que importantes y merecen su tratamiento exclusivo que, sin embargo, a falta de una indagación más cualitativa al respecto, no abordamos en este análisis.

El procedimiento metodológico fue el siguiente:

1. Se realizó en primer lugar un análisis de tablas de contingencia con el fin de observar el nivel de asociación entre las variables cualitativas intervinientes en el análisis y la variable dependiente. Las tablas de contingencia se pueden considerar como un instrumento de carácter fundamental para analizar variables cualitativas y le proporcional al analista social una técnica de lectura rápida de las relaciones entre fenómenos. Con ella se trata de determinar si existe relación (asociación) entre las variables, y cómo es esta relación, o si por el contrario no se da y podemos hablar de independencia entre las variables. Si existe asociación, entonces podrá medirse la fuerza de esa asociación con el índice $\mathrm{V}$ de Cramer que varía entre 0 y 1 marcando ausencia de fuerza o máxima fuerza en esa asociación?.

2. En segundo lugar se aplicó un test de medias para analizar la relación entre las variables cuantitativas (edad y cantidad de hermanos) y la variable dependiente. El

9. En los fenómenos sociales la fuerza de asociación medida con el índice de V de Cramer rara vez pasan valores de 0,6. 
contraste de dos medias consiste en comparar los resultados obtenidos en la estimación de las medias de una variable a partir de muestras aleatorias y ver si existen diferencias entre ambas. A su vez, se busca determinar si esas diferencias son significativas o simplemente se deben al azar.

3. En tercer lugar, como análisis explicativo se utiliza la regresión logística binaria, que permite analizar una variable dependiente $Y_{i}$ que incluye dos alternativas excluyentes en términos de probabilidades (en nuestro caso desarrollar o no actividad de tipo agropecuaria), a partir de las variables independientes. La regresión logística permite pronosticar la pertenencia al grupo a partir de una variable dependiente o categórica cualitativa que en el caso particular de la binaria incluye dos valores. Se trata de una técnica estadística multivariable destinada al análisis de una relación de dependencia, en forma similar al análisis de la regresión lineal. A diferencia de ésta, no se exige que todas las variables sean cuantitativas, sino que se pueden utilizar tanto variables cuantitativas como variables cualitativas (LÓPEZ-ROLDÁN y FACHELLI, 2015).Se sigue una estrategia de modelización sustantiva (JOVELL, 1995), es decir, se incluye en el modelo de análisis aquellas variables que se consideran pertinentes para explicar el fenómeno, de acuerdo al objetivo del trabajo. La otra estrategia (legítima en este tipo de técnicas) es la modelización estadística, que es aquella que solo admite en el modelo variables independientes que tienen una capacidad de predicción estadísticamente significativa. La especificación empírica de las hipótesis previamente presentadas se puede expresar como: donde $P$ es la probabilidad de desarrollar actividades agropecuarias. Se utiliza un vector común de variables independientes y de control para poner a prueba ambas hipótesis. La ecuación 1 representa el vector que contiene la variable independiente, es decir, "Familias que desarrollan actividades agropecuarias (product)" más el conjunto de variables de control, tales como lugar de residencia, sexo, la edad, el nivel de educación, el tipo de estudio y los ingresos. La ecuación 1 se estima mediante el método de máxima verosimilitud (maximum likelihood method) que busca maximizar la probabilidad de ocurrencia de $Y$ dados unos determinados valores de las variables independientes.

Los datos a partir de los cuales se construyó el modelo de análisis fueron obtenidos de una encuesta representativa de 400 jóvenes que residen en diferentes zonas rurales de la Argentina. En su diseño se han utilizado indicadores estándares para la caracterización de los jóvenes tomando como referencia esencial estudios y relevamientos previos sobre la situación en Argentina y otros países de la región (INSTITUTO MEXICANO DE LA JUVENTUD, 2002; ROMÁN, 2003; SAGPyA, 2005; CPJ, 2008; INJUV, 2010; DEL RÍO ALONSO, 2012;). La encuesta ha incorporado entre otros aspectos la situación educativa, laboral, los ingresos, el acceso a la tierra y las perspectivas de futuro como ejes centrales de la investigación.

Las encuestas se han aplicado a una muestra representativa de la población, de entre 16 y 35 años, que reside en territorios rurales dispersos (campo), en parajes rurales y en localidades de menos de 10.000 habitantes con estrecha vinculación al sector agropecuario. La muestra se aplicó en ocho provincias representativas de las principales economías regionales de Argentina (Buenos

$\ln \left\{\frac{P(Y=1)}{1-P(Y=1)}\right\}=\alpha+\beta_{1}$ product $_{\text {si }}+\beta_{2}$ Sexo $_{\text {zarón }}+\beta_{3}$ edad $+\ldots$ 
Aires, Santa Fe, Chaco, Entre Ríos, Corrientes, Misiones, Córdoba y Mendoza). La Tabla 1 y la Figura 2 presentan la selección final de los casos y su distribución, siendo las provincias de Córdoba y Buenos Aires donde se encuentra la mayor cantidad de encuestas realizadas, de acuerdo a la mayor proporción de habitantes que poseen dichas provincias.

El criterio de selección de los encuestados fue en dos etapas. En un primera etapa se seleccionaron las viviendas, y en una segunda etapa el joven dentro de cada vivienda. La elección de las viviendas se realizó mediante la selección de rutas aleatorias. Para ello existieron dos estrategias de abordaje. Por un lado, en los pueblos y parajes se comenzó por el extremo noreste del plano, estableciendo un recorrido de ruta aleatoria hasta cubrir la cuota del perfil del joven rural. Por otro, en la zona estrictamente rural se seleccionaron los establecimientos o las viviendas que se encontraban a ambos lados de la vía, dada la escasez de viviendas y establecimientos rurales en el territorio. Por su parte para la selección del

Tabla 1. Distribución de la muestra por provincia

\begin{tabular}{lcc}
\hline \multicolumn{1}{c}{ Provincia } & $\begin{array}{c}\text { Total de jóvenes en } \\
\text { zonas rurales }\end{array}$ & $\begin{array}{c}\text { Encuestas } \\
\text { realizadas }\end{array}$ \\
\hline Buenos Aires & 257.089 & 78 \\
Chaco & 109.602 & 33 \\
Córdoba & 269.845 & 82 \\
Corrientes & 84.788 & 26 \\
Entre Ríos & 100.292 & 30 \\
Mendoza & 154.259 & 47 \\
Misiones & 129.146 & 39 \\
Santa Fe & 216.580 & 66 \\
\hline Provincias seleccionadas & $\mathbf{1 . 3 2 1 . 6 0 1}$ & $\mathbf{4 0 0}$ \\
\hline
\end{tabular}

Fuente: Elaboración propia sobre la base del Censo de Población, Hogares y Viviendas 2010. joven dentro de cada vivienda (se propuso la realización de solo una encuesta por hogar) se diseñó un mecanismo de selección aleatoria a partir de la tabla de Kish.

El mecanismo de selección del individuo basado en la tabla de Kish requiere los siguientes pasos: 1) Determinar en $n^{\circ}$ de hogar encuestado (selección de la fila); 2) Excluir a las personas que no serán tenida en cuenta para la encuesta, dado que la población objetiva son los jóvenes de 16 a 35 años; 3) Ordenar los jóvenes posibles de ser seleccionados según edad y sexo, enumerando a los jóvenes de menor a mayor (selección de la columna); 4) De acuerdo al ${ }^{\circ}$ de vivienda (fila) y a la cantidad de jóvenes (columna) se selecciona el joven que será encuestado.

A la derecha presenta una simplificación de la tabla de Kish utilizada para la selección de los jóvenes.

\begin{tabular}{|l|c|c|c|c|}
\hline \multirow{2}{*}{\multicolumn{1}{|c|}{$\mathbf{N}^{\circ}$ de hogar }} & \multicolumn{5}{|c|}{$\mathbf{N}^{\circ}$ de jóvenes: } \\
\cline { 2 - 5 } & $\mathbf{1}$ & $\mathbf{2}$ & $\ldots$ & $\mathbf{1 0}$ o más \\
\hline 1 о 11 о 21 о $\ldots$ & 1 & 1 & & 9 \\
\hline 2 о 12 о 22 о ... & 1 & 2 & & 10 \\
\hline$\ldots$ & & & & \\
\hline 10 о 20 о 31 о ... & 1 & 2 & & 8 \\
\hline
\end{tabular}

Figura 2. Distribución geográfica de la muestra

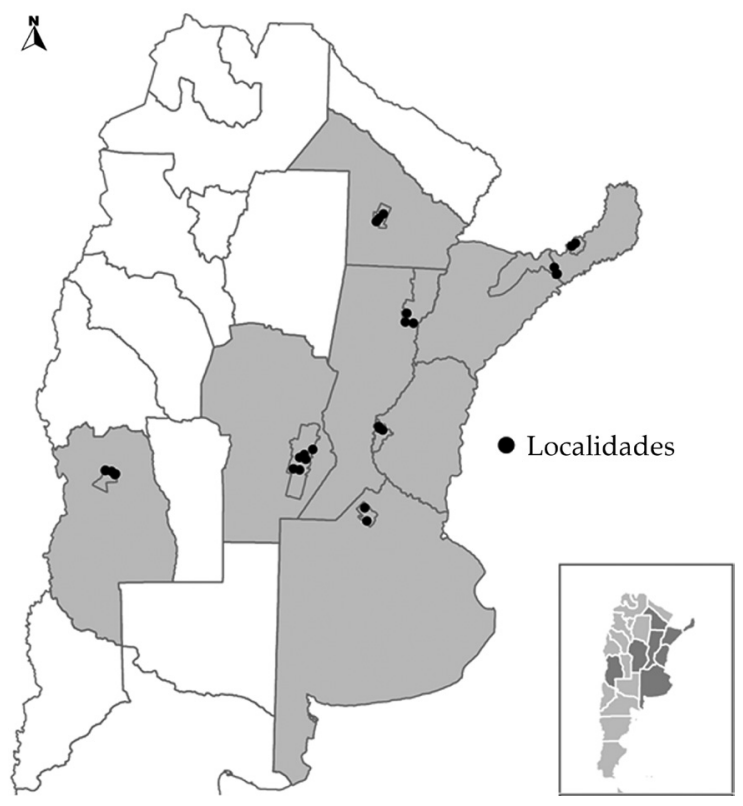

Fuente: Elaboración propia. 
El tamaño de la muestra de 400 casos fue definido considerando un equilibrio entre los recursos disponibles y la posibilidad de obtener un error muestral aceptable para el objetivo del estudio. Considerando un muestreo aleatorio simple para la estimación de una proporción de una población infinita y considerando un nivel de confianza del 95,5\% en el supuesto de máxima varianza o indeterminación $(P=Q=50 \%)$, se obtuvo un error muestral del 5\% para datos globales, según se deriva de la siguiente fórmula:

$$
n=\frac{z^{2} \cdot P \cdot Q}{e^{2}}
$$

donde,

$n$ es el tamaño muestral (400 hogares)

$P \cdot Q$ es la varianza de una proporción, con $Q=$ $100-P$, y $P=Q=50 \%$

$e$ es el error muestral $(5,0 \%)$

$z$ es el número de unidades de desviación, e igual a 2 considerando un nivel de confianza del 95,5\%.

\section{Resultados}

Las tablas de contingencia elaboradas permiten analizar en primer lugar las características generales de las variables categóricas incluidas en el estudio. Las primeras columnas de la Tabla 2 hacen referencia al porcentaje de jóvenes que realizan o no actividades agropecuarias, detallando el total relativo y caracterizándolos según cada una de las variables seleccionadas en el modelo de análisis. A continuación se presenta el test $\mathrm{Chi}^{2}$ que informa si existe relación entre las distintas variables y el hecho de desarrollar o no actividades agropecuarias. Además se presenta un índice que muestra qué tan fuerte resulta esa asociación (V de Cramer). Dicho índice varía entre 0 y 1 mostrando el 1 un grado máximo de asociación, difícilmente encontrado en las variables sociales (LÓPEZ-ROLDÁN y FACHELLI, 2015).

Tal como se observa en la Tabla 2, las variables independientes tienen asociación significativa con la variable dependiente, excepto proyecto productivo. Todas las que presentan asociación, excepto la educación y el hecho de que algún miembro de la familia sea cooperativista, alcanzan los mayores niveles de significación estadística (hasta el 0,01).

Por otro lado al analizar la fuerza de la asociación entre las variables observamos que tener o no estudios agropecuarios en primer lugar, el sexo, el hecho que su familia es productora agropecuaria y la ubicación de la vivienda son las que más fuerza tienen con el hecho de que los jóvenes desarrollen actividades agropecuarias cuando se analiza cada uno de estos aspectos en forma individual. Por su parte el pertenecer a una cooperativa así como el nivel de estudios de los jóvenes conserva relación con el hecho de desarrollar una actividad agropecuaria pero esa relación es más débil (0,18 y 0,16 respectivamente). Por último, se vuelve a señalar que el hecho de desarrollar un proyecto productivo no presenta una asociación significativa con la variable dependiente.

El test de medias (T-Student) realizado en segundo lugar sirva para analizar si hay diferencias significativas en la edad de los jóvenes que desarrollan actividades agropecuarias y los que no, arroja los resultados en la Tabla 3.

La diferencia de edad entre los que desarrollan actividades agropecuarias (promedio de 25,6 años) y los que no desarrollan actividades agropecuarias (promedio de 23 años) es significativa, con lo cual podemos afirmar que son los jóvenes que tienen mayor edad los que están más inclinados a realizar dichas actividades.

Por su parte la otra variable cuantitativa que interviene en el análisis es la cantidad de hermanos. La idea es constatar si existe una correlación positiva entre poseer mayor cantidad de hermanos y el desarrollo de una actividad agropecuaria. El 88\% de los jóvenes encuestados tienen entre 1 y 7 hermanos, siendo 1,8 el número promedio total de hermanos. No obstante el test de medias nos informa que no existe relación entre el hecho de poseer hermanos y el de desarrollar una actividad agropecuaria (Tabla 4). 
Tabla 2. Resultado de los cruces entre variable dependiente e independientes

\begin{tabular}{|c|c|c|c|c|c|c|c|}
\hline \multirow[t]{2}{*}{ Variables } & \multicolumn{3}{|c|}{$\begin{array}{c}\text { Desarrollo de Actividad } \\
\text { Agropecuaria Propia }\end{array}$} & \multicolumn{3}{|c|}{$\mathrm{Chi}^{2}$} & \multirow{2}{*}{$\begin{array}{c}\text { Fuerza de la } \\
\text { Asoc. (V de } \\
\text { Cramer) }\end{array}$} \\
\hline & Sí & No & Total & Valor & Sig & Sig. & \\
\hline Ubicación de la vivienda & & & & 27,33 & 0,00 & $* *$ & 0,26 \\
\hline Disperso & $61,9 \%$ & $28,0 \%$ & $33,4 \%$ & & & & \\
\hline Agrupado & $38,1 \%$ & $72,0 \%$ & $66,6 \%$ & & & & \\
\hline Sexo & & & & 31,71 & 0,00 & $* *$ & 0,28 \\
\hline Varón & $87,3 \%$ & $48,8 \%$ & $54,9 \%$ & & & & \\
\hline Mujer & $12,7 \%$ & $51,2 \%$ & $45,1 \%$ & & & & \\
\hline Nivel de estudios & & & & 10,03 & 0,01 & $* *$ & 0,16 \\
\hline Bajo & $11,3 \%$ & $6,3 \%$ & $7,1 \%$ & & & & \\
\hline Medio & $61,3 \%$ & $44,9 \%$ & $47,5 \%$ & & & & \\
\hline Alto & $27,4 \%$ & $48,8 \%$ & $45,4 \%$ & & & & \\
\hline Estudios agropecuarios & & & & 36,96 & 0,00 & $* *$ & 0,30 \\
\hline $\mathrm{Si}$ & $58,7 \%$ & $21,5 \%$ & $27,4 \%$ & & & & \\
\hline No & $41,3 \%$ & $78,5 \%$ & $72,6 \%$ & & & & \\
\hline Ingreso total (rango) & & & & 14,59 & 0,00 & $* *$ & 0,19 \\
\hline Hasta $\$ 5.000$ & $39,7 \%$ & $54,8 \%$ & $52,4 \%$ & & & & \\
\hline Más de $\$ 5.000$ & $46,0 \%$ & $22,9 \%$ & $26,6 \%$ & & & & \\
\hline No declara & $14,3 \%$ & $22,3 \%$ & $21,0 \%$ & & & & \\
\hline Su familia es productora agropecuaria & & & & 30,14 & 0,00 & $* *$ & 0,27 \\
\hline Sí & $93,7 \%$ & $57,2 \%$ & $63,0 \%$ & & & & \\
\hline No & $6,3 \%$ & $42,8 \%$ & $37,0 \%$ & & & & \\
\hline Pertenecen a alguna cooperativa agropecuaria & & & & 12,86 & 0,00 & $* *$ & \\
\hline Sí & $63,5 \%$ & $44,9 \%$ & $47,9 \%$ & & & & 0,18 \\
\hline No, pero con relación & $14,3 \%$ & $8,6 \%$ & $9,5 \%$ & & & & \\
\hline No cooperativista & $22,2 \%$ & $46,5 \%$ & $42,5 \%$ & & & & \\
\hline Proyecto propio & & & & 3,54 & 0,06 & ns & -- \\
\hline Sí & $19,6 \%$ & $30,2 \%$ & $21,3 \%$ & & & & \\
\hline No & $80,4 \%$ & $69,8 \%$ & $78,7 \%$ & & & & \\
\hline Total & $100 \%$ & $100 \%$ & $100 \%$ & & & & \\
\hline
\end{tabular}

${ }^{*}$ Coeficiente significativo inferior al 0,$05 ;{ }^{* *}$ Coeficiente significativo al 0,$01 ;$ ns Coeficiente no significativo.

Fuente: Elaboración propia.

Tabla 3. Test de medias para la variable Edad

\begin{tabular}{|c|c|c|c|c|c|c|c|c|}
\hline \multirow{2}{*}{\multicolumn{2}{|c|}{$\begin{array}{l}\text { Prueba de muestras } \\
\text { independientes }\end{array}$}} & \multicolumn{2}{|c|}{$\begin{array}{c}\text { Prueba de Levene } \\
\text { para la igualdad de } \\
\text { varianzas }\end{array}$} & \multicolumn{5}{|c|}{ Prueba $\mathrm{T}$ para la igualdad de medias } \\
\hline & & $F$ & Sig. & $t$ & $g l$ & $\begin{array}{c}\text { Sig. } \\
\text { (bilateral) }\end{array}$ & $\begin{array}{l}\text { Diferencia de } \\
\text { medias }\end{array}$ & $\begin{array}{c}\text { Error típ. de la } \\
\text { diferencia }\end{array}$ \\
\hline \multirow{2}{*}{$\begin{array}{l}\text { Edad } \\
\text { (entre 16 y } \\
35 \text { años) }\end{array}$} & $\begin{array}{l}\text { Se han asumido } \\
\text { varianzas iguales }\end{array}$ & 0,10 & 0,74 & $-3,63$ & 393 & 0,00 & $-2,59$ & 0,71 \\
\hline & $\begin{array}{l}\text { No se han asumido } \\
\text { varianzas iguales }\end{array}$ & & & $-3,71$ & 88,90 & 0,00 & $-2,59$ & 0,69 \\
\hline
\end{tabular}

Fuente: Elaboración propia.

Tabla 4. Test de medias para la cantidad de Hermanos

\begin{tabular}{|c|c|c|c|c|c|c|c|c|}
\hline \multirow{2}{*}{\multicolumn{2}{|c|}{$\begin{array}{l}\text { Prueba de muestras } \\
\text { independientes }\end{array}$}} & \multicolumn{2}{|c|}{$\begin{array}{l}\text { Prueba de Levene } \\
\text { para la igualdad de } \\
\text { varianzas }\end{array}$} & \multicolumn{5}{|c|}{ Prueba $T$ para la igualdad de medias } \\
\hline & & $F$ & Sig. & $t$ & $g l$ & $\begin{array}{c}\text { Sig. } \\
\text { (bilateral) }\end{array}$ & $\begin{array}{l}\text { Diferencia de } \\
\text { medias }\end{array}$ & $\begin{array}{c}\text { Error típ. de la } \\
\text { diferencia }\end{array}$ \\
\hline \multirow{2}{*}{$\begin{array}{l}\text { Cantidad de } \\
\text { hermanos }\end{array}$} & $\begin{array}{l}\text { Se han asumido } \\
\text { varianzas iguales }\end{array}$ & 2,43 & 0,11 & 0,43 & 393 & 0,66 & 0,08 & 0,18 \\
\hline & $\begin{array}{l}\text { No se han asumido } \\
\text { varianzas iguales }\end{array}$ & & & 0,49 & 100,82 & 0,62 & 0,08 & 0,16 \\
\hline
\end{tabular}

Fuente: Elaboración propia. 
646 Juventud Rural: factores que influyen en el desarrollo de la actividad agropecuaria. Reflexiones sobre el caso argentino

Más allá de este análisis inicial, es la regresión logística binaria la que definitivamente permite evaluar desde una perspectiva general y más sistémica la incidencia o el efecto de cada una de las variables, teniendo en cuenta el resto de las características de los jóvenes. Los resultados de la regresión logística que presenta una capacidad explicativa del 42\% (R cuadrado de Nagelkerke 0,42 ) se presentan a continuación (Tabla 5).

Tabla 5. Resultados de la Regresión logística binaria

\begin{tabular}{|c|c|c|c|c|c|}
\hline \multicolumn{6}{|c|}{ Modelo Desarrolla Actividad Agropecuaria Propia } \\
\hline $\begin{array}{l}\text { V. Dependiente: Desarrolla Actividad } \\
\text { Categ. De referencia: Sí }\end{array}$ & B & Error típico & $\operatorname{Exp}(b)$ & Sig. & Sig. \\
\hline \multicolumn{6}{|l|}{ Ubicación de la vivienda: Agrupado $\left(^{*}\right)$} \\
\hline Disperso & 1,14 & 0,35 & 3,12 & 0,00 & ** \\
\hline \multicolumn{6}{|l|}{ Sexo: Mujer $\left({ }^{*}\right)$} \\
\hline Varón & 1,38 & 0,47 & 3,98 & 0,00 & ** \\
\hline Edad & 0,09 & 0,04 & 1,09 & 0,02 & * \\
\hline Cantidad de hermanos & $-0,12$ & 0,14 & 0,89 & 0,42 & ns \\
\hline Nivel de estudios agrupados Bajo $\left({ }^{*}\right)$ & 0,00 & 0,00 & 0,00 & 0,05 & ns \\
\hline Medio & $-0,10$ & 0,62 & 0,90 & 0,87 & ns \\
\hline Alto & $-1,08$ & 0,67 & 0,34 & 0,11 & ns \\
\hline \multicolumn{6}{|l|}{ Estudios agropecuarios: No $\left.{ }^{*}\right)$} \\
\hline Sí & 0,98 & 0,38 & 2,66 & 0,01 & ** \\
\hline Ingreso total (rango): Hasta $\$ 5.000\left(^{*}\right)$ & 0,00 & 0,00 & 0,00 & 0,09 & ns \\
\hline Más de $\$ 5.000$ & 0,87 & 0,40 & 2,39 & 0,03 & * \\
\hline No declara Ingreso & 0,12 & 0,51 & 1,12 & 0,82 & ns \\
\hline \multicolumn{6}{|l|}{ Su familia es productora agropecuaria: Familia NO productora $\left({ }^{*}\right)$} \\
\hline Familia productora & 2,00 & 0,59 & 7,37 & 0,00 & ** \\
\hline Pertenecen a alguna cooperativa agropecuaria: Cooperativista $\left(^{*}\right)$ & & & & 0,78 & ns \\
\hline No, pero con relación & $-0,37$ & 0,52 & 0,69 & 0,48 & ns \\
\hline No cooperativista & $-0,11$ & 0,43 & 0,90 & 0,81 & ns \\
\hline \multicolumn{6}{|l|}{ Proyecto propio: Sí $\left({ }^{*}\right)$} \\
\hline No & 0,48 & 0,40 & 1,61 & 0,23 & ns \\
\hline Constante & $-7,27$ & 1,42 & 0,00 & 0,00 & ** \\
\hline Número de casos & 392,00 & & & & \\
\hline-2 log de la verosimilitud & 228,45 & & & & \\
\hline R cuadrado de Nagelkerke & 0,42 & & & & \\
\hline Prueba de Hosmer y Lemeshow & 0,15 & & & & \\
\hline Valor pronosticado inicial & 84,40 & & & & \\
\hline Valor pronosticado final & 87,80 & & & & \\
\hline
\end{tabular}

$\left({ }^{*}\right)$ Categoría de Referencia de las variables independientes.

** Coeficiente significativo al 0,01.

* Coeficiente significativo inferior al 0,05.

ns Coeficiente no significativo.

Nota: Se describe a continuación algunos términos.

B: Significación de la variable independiente. Si es menor de 0,05 esa variable independiente explica la variable dependiente.

$\operatorname{Exp}(b)$ : Exponencial de b indica la fortaleza de la relación. Cuanto más alejada de 1 está más fuerte es la relación.

Fuente: Elaboración propia. 


\section{Discusión de los resultados}

El análisis de regresión logística permite confirmar nuestra hipótesis que plantea que pertenecer a una familia productora es el factor que explica en mayor medida que el joven desarrolle actividades agropecuarias y permanezca en las zonas rurales. Así, ser parte de una familia productora (familia con EAP) multiplica por 7 la probabilidad de desarrollar una actividad agropecuaria, un resultado muy contundente que muestra cómo la construcción de un ambiente sociofamiliar en donde prima un aprendizaje cotidiano y una participación activa desde niño en las actividades agropecuarias es el factor más importante en la transmisión de conocimientos y en la adquisición de hábitos agropecuarios que favorecen la instalación y permanencia del joven en el medio rural, realizando actividades agropecuarias. Este resultado afirma y consolida la línea de reflexión que pone énfasis en la dimensión social e identitaria de los jóvenes, tal como lo plantea en términos generales White (2012) y Dirven (1995), o Costa y Ralisch (2013) en el caso brasilero y Jurado y Tobasura (2012) en el caso de Colombia. Los datos de la encuesta muestran además que los jóvenes que han desarrollado una actividad agropecuaria provienen en su gran mayoría de familias agropecuarias $(93,7 \%)$, que trabajan o tienen explotaciones medias. Además el análisis nos muestra que son pocos los casos de jóvenes que quieren emprender una actividad agropecuaria cuyas familias son no agropecuarias $(6,3 \%)$.

Con respecto al sexo, la regresión muestra que los hombres tienen cuatro veces más probabilidades de desarrollar una actividad agropecuaria si se compara con las mujeres, las cuales continúan en mayor mediad sus estudios especialmente en actividades no agropecuarias. En términos porcentuales, de los jóvenes que han desarrollado una actividad agropecuaria el $87 \%$ son varones, y el 13\% mujeres (cuando la muestra está equilibrada en términos de género), aún más, a medida que aumenta la edad de las mujeres, su continuidad en el medio rural y en torno a una actividad agropecuaria disminuye sensible- mente. En realidad, como hemos comentado, no realizamos un estudio de género, por ello dejamos pendiente problematizar estos resultados. Hace falta reflexionar sobre los mecanismos que impiden a las mujeres heredar o ser recluidas al ámbito privado o de cuidado, y de esa manera, estos resultados nos permiten hipotetizar sobre la posibilidad de que el estudio sea una opción forzada de las mujeres ante la preferencia familiar de que sean varones los herederos (CASTRO et al., 2015).

El lugar de residencia de los jóvenes también es de gran importancia. Los resultados muestran que los jóvenes que viven en el campo tienen tres veces más probabilidades de desarrollar una actividad agropecuaria que otros jóvenes que viven en el pueblo, es decir, es la vida en una explotación agropecuaria lo que permite fortalecer una identidad agropecuaria que favorece la construcción de un proyecto de vida y de trabajo en el campo.

Según el modelo planteado, existe una relación importante entre el hecho de que los jóvenes realicen estudios agropecuarios y que desarrollen una actividad agropecuaria. Así las escuelas agrotécnicas y agropecuarias en general contribuyen a consolidar una identidad agropecuaria y a formar a los jóvenes en temas agropecuarios que ya orientan a los jóvenes a dicha actividad en el campo. Estos mismos resultados también fueron planteados por la SAGPyA (2005) para el caso argentino en general.

El nivel educativo de los jóvenes fue otro factor analizado, pues generalmente se presume que el mismo tiene algún tipo de influencia sobre la continuidad en el campo y en la actividad agropecuaria. Sin embargo los resultados obtenidos permiten observar que el nivel de estudios no tiene una correlación directa con la permanencia de los jóvenes en el campo. Una primera lectura de los datos porcentuales muestran que el $61 \%$ de los jóvenes agricultores tienen niveles medios de formación, en tanto sólo el 27\% tienen niveles altos de formación. Comparado con los jóvenes que no poseen una actividad agropecuaria el $45 \%$ posee estudios medios y el $49 \%$ estudios altos. 
No obstante la regresión muestra que la realización de mayores niveles de estudio no tiene relación directa con la realización de actividades agropecuarias

Por último, partimos de la premisa que para el joven tiene importancia las cooperativas y las asociaciones de productores como ámbito de construcción de la identidad y el oficio de productor agropecuario (SILI et al., 2014). Al analizar la relación que se establece entre los jóvenes agricultores y la participación de sus familias en asociaciones o cooperativas se observa que el 63\% de los agricultores tienen un vínculo muy importante con las entidades cooperativas, ya sea porque sus padres están asociados o porque existe una relación comercial habitual. Este es el resultado cuando se analiza el nivel de asociación con la variable dependiente. No obstante, cuando se toman en cuenta todas las variables del modelo propuesto, queda de manifiesto que ser hijos de padres cooperativistas no incentiva a los jóvenes a desarrollar su propia actividad agropecuaria ${ }^{10}$. En ese sentido la participación de las familias en grupos cooperativos o en asociaciones de productores no constituiría un factor clave en la permanencia de los jóvenes en el medio rural y en la actividad agropecuaria

\section{Conclusión}

El análisis realizado permite concluir claramente que son los jóvenes varones, que viven en el campo, que son hijos de productores agropecuarios y que han realizado estudios agropecuarios los que tienen una mayor probabilidad de desarrollar una actividad agropecuaria propia. Claramente es el tipo de hábitat y el contexto familiar en el cual los jóvenes crecieron y

10. Este resultado ha sido corroborado al hacer pruebas adicionales cambiando la categoría de aquellos que no participan en cooperativas pero tienen algún tipo de relación comercial con ellas, tanto sumándola a la categoría Participa como a la categoría No participa, de manera de dicotomizar la variable. Los resultados obtenidos son los mismos, la variable no resulta significativa al ser controlada por el resto de las variables independientes. se educaron el que permite construir hábitos y una fuerte identidad por la actividad agropecuaria y por el medio rural, es decir, se trata de factores que podemos denominar "socioambientales", en donde el peso de las condiciones territoriales (vivir en el campo) y productivas (dedicación de la familia a la actividad agropecuaria) son relevantes.

De esta manera este trabajo permite observar que la construcción de una trayectoria de vida en el medio rural y en el campo, ligado a una actividad agropecuaria, y a una educación vinculada al campo, es lo determinante que un joven construya un proyecto de vida en el mismo. Las condiciones socioambientales en donde el joven desarrolla su vida son claramente definitorias y favorecen la continuidad en el lugar. Desde una perspectiva teórica y conceptual, esta conclusión refuerza claramente la línea de reflexión de Dirven (1995), Sili (2005b), White (2012), Jurado (2012), planteada al inicio de este trabajo, centrada en las condiciones sociales y ambientales constructoras de la identidad como factores claves de la permanencia de los jóvenes en el medio rural realizando actividades agropecuarias. Esta línea argumental pone claramente énfasis en factores sociales y culturales antes que en factores que a priori aparecen como más estructurales como son el nivel de acumulación o la posesión de tierras.

Estos hallazgos tienen profundas implicancias en términos de políticas públicas para el desarrollo agropecuario y rural. Una política que pretenda consolidar el arraigo y la permanencia de los jóvenes en el medio rural desarrollando actividades agropecuarias debería poner el acento en fortalecer y apoyar económicamente la estructura sociofamiliar de aquellas familias que ya realizan dichas tareas en pos de garantizar la trayectoria de los jóvenes hacia la actividad laboral agropecuaria, así como también promover los estudios vinculados a la producción agropecuaria. Finaliemnte, consideramos que son necesarios otros estudios para profundizar los hallazgos obtenidos, para continuar explorando más elementos vinculados a los aquí estudiados, como 
por ejemplo un análisis de género para poder comprender el proceso de exclusión de la mujer en el ámbito rural y para delinear con mayor precisión las políticas públicas necesarias en pos de estimular y favorecer el desarrollo de la actividad agropecuaria por parte de los jóvenes que viven en zonas rurales.

\section{Referencias}

BAJEMA, D. MILLER, W. y WILLIAMS, D. Aspirations of rural youth. Journal of Agricultural Education, v. 43, n. 3, p. 61-71, 2002.

BARCELLOS, S. A Formulação das políticas públicas para a juventude rural no Brasil e os elementos constitutivos desse processo social. Mundo Agrario. Revista de Estudios Rurales, v. 16, n. 32, p. 4-32, 2015.

CASTRO, A. M. P. PEREZ, M. V. FERRE, M. B. RAMON, M. D. G. y SERRA, I. S. Formación de las mujeres, empoderamiento e innovación rural. Boletín de la Asociación de Geógrafos Españoles, n. 68, p. 385-406, 2015.

COSTA, F. L. M. y RALISCH, R. A. Juventude rural do assentamento Florestan Fernandes no município de Florestópolis (PR). Revista de Economía e Sociología Rural, v. 51, n. 3, p. 415-432, 2013.

COTA MENDONÇA, K. F., RIBEIRO, E. y GALIZONI, F. Formação, sucessão e migração: trajetórias de duas gerações de agricultores do Alto Jequitinhonha, Minas Gerais. Revista Brasileira de Estudos de População, v. 30, n. 2, p. 455-463, 2013.

CPJ. Primera Encuesta Nacional de Juventud, Costa Rica. Consejo Nacional de la Política Pública de la Persona Joven, Observatorio de la Persona Joven, 1a ed., San José, Costa Rica: Fondo de Población de las Naciones Unidas, UNFPA, 2008.

CREED, G. y CHING, B. Recognition Rusticity. Identity and the power of place. In: CREED, G. y CHING, B. (Ed.). Knowing your place. Rural identity and cultural hierarchy. New York: Routledge, 1997.

DE MOLINA NAVARRO, M. L. G. La cuestión agraria en la historia de Andalucía: nuevas perspectivas, 2014.

DEL RÍO ALONSO, J. Diagnóstico de la situación de la juventud de Canarias. Encuesta de Jóvenes de Canarias. Técnicos en Socioanálisis, Gobierno de Canaria, 2012.

DIRVEN, M. Expectativas de la juventud y el desarrollo rural. Revista CEPAL, Santiago de Chile, n. 55, p. 123138, 1995.
. Las prácticas de herencia de tierras agrícolas: ¿una razón más para el éxodo de la juventud?. Revista CEPAL, Serie de Desarrollo Productivo, Santiago de Chile, n. 135, p. $24,2002$.

DURSTON, J. Juventud y desarrollo rural: Marco conceptual y contextual. Revista CEPAL, Documentos de Trabajo, Santiago de Chile, n. 28, p. 22, 1998.

FACHELLI, S. y LÓPEZ-ROLDÁN, P. Análisis de Datos Estadísticos. Análisis de Movilidad Social. Departamento de Sociología, Universidad Autónoma de Barcelona, Bellaterra, 2013. Disponible en: < http://ddd.uab.cat/ pub/recdoc/2012/88747/ADEAnalisisMovilidadSocial_ a2013.pdf $>$.

GARRABOU, R., PLANAS, J., SAGUER, E. y VICEDO, E. Propiedad de la tierra y desigualdad social en el mundo rural catalán de mediados del siglo XIX. Historia agraria: revista de agricultura e historia rural, n. 63, p. 47-82, 2014.

INDEC. Censo Nacional de Población, Hogares y Viviendas, 2010.

INJUV. Sexta Encuesta Nacional de Juventud. Instituto Nacional de la Juventud, Gobierno de Chile, 2010.

INSTITUTO MEXICANO DE LA JUVENTUD. Encuesta Nacional de Juventud 2000. Secretaría de Educación Pública, Instituto Mexicano de la Juventud, Centro de Investigación y Estudios sobre Juventud. México, D.F., 2002.

JOVELL, A. Análisis de regresión logística. Cuadernos Metodológicos del CIS, Madrid, n. 15, 1995.

JURADO, C. y TOBASURA, I. Dilema de la juventud en territorios rurales de Colombia: ¿campo o ciudad? Revista Latinoamericana de Ciencias Sociales, Niñez $Y$ Juventud, v. 10, n. 1, p. 63-77, 2012.

KESSLER, G. Estado del arte de la investigación sobre juventud rural en América Latina (p. 43). Universidad Nacional General Sarmiento, Argentina, 2005.

LINCK, T. El campo en la ciudad: reflexiones en torno a las ruralidades emergentes. En Memorias del seminario internacional "La nueva ruralidad en América Latina". Bogotá: Pontificia Universidad Javeriana, 2001.

LOPES, L. G. R. y CARVALHO, D. B. Dinâmica Temporal do Assentamento e os Projetos de Vida da Juventude Rural. Revista de Economía e Sociología. Rural, v. 53, n. 2, p. 571-588, 2015.

LÓPEZ-ROLDÁN, P. y FACHELLI, S. Metodología de la Investigación Social Cuantitativa. Bellaterra (Cerdanyola del Vallès): Dipòsit Digital de Documents, Universitat 
Auònoma de Barcelona, 2015. Disponible en: <http:// ddd.uab.cat/record/129382>.

MAURICIO, R. Trayectorias laborales de los jóvenes en Argentina: ¿Dificultades en el mercado de trabajo o carrera laboral ascendente?. Revista CEPAL, Serie Macroeconomía del desarrollo, Santiago de Chile, n. 109, p. 83, 2011.

PACHECO LADRÓN DE GUEVARA, L. Nueva ruralidad y empleo. El reto de la educación de los jóvenes rurales en América Latina. Cuadernos de Desarrollo Rural, n. 43, 1999.

ROMÁN, M. Los jóvenes y las jóvenes rurales en Argentina. Elementos para una estrategia de desarrollo rural. SAGPyAPROINDER, Serie de Estudios e Investigaciones, Buenos Aires, 2003.

SAGPyA. Educación, desarrollo rural y juventud. Convenio de Cooperación Secretaría de Agricultura Ganadería, Pesca y Alimentos de la Nación e Instituto Internacional de Planeamiento de la Educación Sede Regional Buenos Aires (IIPE - UNESCO, Buenos Aires), Buenos Aires, 2005.

SARAVÍ, G. Juventud y sentidos de pertenencia en América Latina: causas y riesgos de la fragmentación social. Revista CEPAL, Santiago de Chile, n. 98, p. 47-65, 2003.

SCHEJTMAN, A. y BERDEGUÉ J. Desarrollo territorial rural. Santiago de Chile. RIMISP, mimeo, 2003.

SILI, M. La Juventud frente a la crisis rural. Revista Universitaria de Geografía, v. 14, n. 1 y 2, p. 137-162, 2005a.
. La Argentina Rural. De la crisis de la modernización agraria a la construcción de un nuevo paradigma de desarrollo de los territorios rurales. Buenos Aires: INTA, 2005b.

. Les espaces vides de la modernisation rurale - Dépeuplement. In: BARTHE, L. et al. (Eds.), Actes du 2 e colloque franco-espagnol de géographie rurale. Clemont Ferrand: CERAMAC, 2007, p. 153-170.

. y SOMOULOU, L. La problemática de la tierra en argentina. Roma: FIDA (Ed.), 2011, p. 253.

., SANGUINETTI, J. y MEILLER, A. El cooperativismo agrario y su contribución al desarrollo local. La experiencia de la Unión Agrícola de Avellaneda, Argentina. Revista de Economía Pública, Social y Cooperativa, n. 82, p. 155-177, 2014.

., GUIBERT, M. y BUSTOS CARA, R. Atlas de la Argentina rural. Buenos Aires: Capital Intelectual, 2015.

SILVA, N. D. V. y KASSOUF, A. L. A exclusão social dos jovens no mercado de trabalho brasileiro. Revista Brasileira de Estudos de População, v. 19, n. 2, p. 99-115, 2002.

THEOBALD, P. y WOOD, K. Learning to Be Rural. Identity lesson from history and schooling. In: SCHAFFT, K. (Ed.). Rural Education for the Twenty first century. Penssylvania State University Press, 1992.

WELLER, J. Los jóvenes y el empleo en América Latina. Desafíos y perspectivas ante el nuevo escenario laboral. Bogota: Mayol Ediciones, CEPAL Ed., 2006, p. 301.

WHITE, B. Agriculture and the Generation Problem: Rural Youth, Employment and the Future of Farming. IDS Bulletin, v. 43, n. 6, p. 9-21, 2012. 
Tanto en Argentina como en América Latina se dirime una profunda discusión entre dos sectores de pensamiento que plantean posiciones antagónicas. Algunos autores plantean que la posesión de la tierra por parte de los jóvenes es indispensable para que puedan continuar en el sector agropecuario y en el medio rural, es lo que puede plantearse como una postura estructuralista, sin embargo, otros sectores plantean que el factor más importante sería la mejora del nivel educativo, una mayor conectividad y servicios (caminos, infraestructuras de comunicación, servicios) y el acceso a redes de apoyo para la generación de proyectos productivos (SILI et al., 2011), lo cual puede plantearse como una postura más constructivista o más vinculada a la idea de la Nueva Ruralidad en América Latina.

En este sentido nuestros resultados, con una aproximación limitada al tema de la tierra, si la familia es poseedora o no de la tierra, abonaría el hallazgo del segundo grupo de autores, toda vez que dicha posesión no influye el desarrollo de una actividad agropecuario por parte de los jóvenes. Así queda reflejado en la significación del coeficiente Beta que es de 0,90 (Tabla A1).

Así, esta investigación también permite observar que la permanencia de los jóvenes en el medio rural y en la actividad agropecuaria no depende directamente de la posesión de tierras por parte de sus familias (con una aproximación rústica al tema). Esto nos lleva a pensar que son deseables políticas que promuevan la creación de oportunidades de empleo o a la generación de actividades productivas con mecanismos más flexible de acceso a la tierra (arrendamiento, contratos temporales, etc.). Claramente esto deja abierta una importante discusión sobre la problemática de la tierra y su tenencia en la Argentina y en América Latina en general y que podría tener fuertes repercusiones en términos de políticas públicas de desarrollo rural. Explorar investigaciones como las de Gonzáles de Molina (2014) o de Garrabou et al. (2014) ayudarán a plantear modelos de análisis interesantes para abordar esta cuestión. 
652 Juventud Rural: factores que influyen en el desarrollo de la actividad agropecuaria. Reflexiones sobre el caso argentino

Tabla A1. Resultados de la Regresión logística binaria incluyendo posesión de tierra

\begin{tabular}{|c|c|c|c|c|c|}
\hline \multicolumn{6}{|c|}{ Modelo Desarrolla Actividad Agropecuaria Propia } \\
\hline $\begin{array}{l}\text { V. Dependiente: Desarrolla Actividad } \\
\text { Categ. De referencia: Sí }\end{array}$ & B & Error típico & $\operatorname{Exp}(b)$ & Sig. & Sig. \\
\hline \multicolumn{6}{|l|}{ Ubicación de la vivienda: Agrupado $\left(^{*}\right)$} \\
\hline Disperso & 1,13 & 0,35 & 3,11 & 0,00 & ** \\
\hline \multicolumn{6}{|l|}{ Sexo: Mujer $\left(^{*}\right)$} \\
\hline Varón & 1,38 & 0,47 & 3,98 & 0,00 & ** \\
\hline Edad & 0,09 & 0,04 & 1,09 & 0,02 & * \\
\hline Cantidad de hermanos & $-0,11$ & 0,15 & 0,89 & 0,45 & ns \\
\hline Nivel de estudios agrupados Bajo $\left({ }^{*}\right)$ & 0,00 & 0,00 & 0,00 & 0,05 & ns \\
\hline Medio & $-0,10$ & 0,62 & 0,90 & 0,87 & ns \\
\hline Alto & $-1,08$ & 0,67 & 0,34 & 0,11 & ns \\
\hline \multicolumn{6}{|l|}{ Estudios agropecuarios: No $\left({ }^{*}\right)$} \\
\hline Sí & 0,98 & 0,38 & 2,67 & 0,01 & $* *$ \\
\hline Ingreso total (rango): Hasta $\$ 5.000\left(^{*}\right)$ & 0,00 & 0,00 & 0,00 & 0,09 & ns \\
\hline Más de $\$ 5.000$ & 0,87 & 0,40 & 2,38 & 0,03 & $*$ \\
\hline No declara Ingreso & 0,11 & 0,52 & 1,11 & 0,84 & ns \\
\hline \multicolumn{6}{|l|}{ Su familia es productora agropecuaria: Familia NO productora $\left({ }^{*}\right)$} \\
\hline Familia productora & 1,96 & 0,67 & 7,08 & 0,00 & ** \\
\hline Pertenecen a alguna cooperativa agropecuaria: Cooperativista $\left({ }^{*}\right)$ & 0,00 & 0,00 & 0,00 & 0,77 & ns \\
\hline No, pero con relación & $-0,37$ & 0,52 & 0,69 & 0,48 & ns \\
\hline No cooperativista & $-0,11$ & 0,43 & 0,89 & 0,79 & ns \\
\hline \multicolumn{6}{|l|}{ Proyecto propio: Sí $\left({ }^{*}\right)$} \\
\hline No & 0,48 & 0,40 & 1,61 & 0,23 & ns \\
\hline \multicolumn{6}{|l|}{ Posesión de tierras en la familia. Sí $\left({ }^{*}\right)$} \\
\hline No & $-0,05$ & 0,42 & 0,95 & 0,90 & ns \\
\hline Constante & $-7,20$ & 1,51 & 0,00 & 0,00 & $* *$ \\
\hline Número de casos & 392 & & & & \\
\hline$-2 \log$ de la verosimilitud & 228,43 & & & & \\
\hline R cuadrado de Nagelkerke & 0,42 & & & & \\
\hline Prueba de Hosmer y Lemeshow & 0,15 & & & & \\
\hline Valor pronosticado inicial & 84,4 & & & & \\
\hline Valor pronosticado final & 87,8 & & & & \\
\hline \multicolumn{6}{|l|}{${ }^{*}$ ) Categoría de Referencia de las variables independientes. } \\
\hline \multicolumn{6}{|l|}{ * Coeficiente significativo inferior al 0,05. } \\
\hline \multicolumn{6}{|l|}{ ** Coeficiente significativo al 0,01. } \\
\hline \multicolumn{6}{|l|}{ ns Coeficiente no significativo. } \\
\hline \multicolumn{6}{|l|}{ Nota: Se describe a continuación algunos términos. } \\
\hline \multicolumn{6}{|c|}{ B: Significación de la variable independiente. Si es menor de 0,05 esa variable independiente explica la variable dependiente. } \\
\hline $\operatorname{Exp}(b)$ : Exponencial de b indica la fortaleza de la relación. Cuanto más aleja & a de 1 má & cuerte es la relac & & & \\
\hline Fuente: Elaboración propia. & & & & & \\
\hline
\end{tabular}

